\title{
Effect of Interval between Neoadjuvant Chemoradiotherapy and Surgery on Oncological Outcome for Rectal Cancer: A Systematic Review and Meta-Analysis
}

\author{
Xiao-Jie Wang, ${ }^{1}$ Zheng-Rong Zheng, ${ }^{2}$ Pan Chi, ${ }^{1}$ Hui-Ming Lin, \\ Xing-Rong Lu, ${ }^{1}$ and Ying Huang ${ }^{1}$ \\ ${ }^{1}$ Department of Colorectal Surgery, Union Hospital, Fujian Medical University, Fuzhou, Fujian 350001, China \\ ${ }^{2}$ Oncology Department, The Second Affiliated Hospital of Fujian Medical University, Quanzhou, Fujian 360000, China \\ Correspondence should be addressed to Pan Chi; chipan363@163.com
}

Received 30 June 2015; Revised 9 November 2015; Accepted 10 November 2015

Academic Editor: Ralf-Dieter Hofheinz

Copyright (c) 2016 Xiao-Jie Wang et al. This is an open access article distributed under the Creative Commons Attribution License, which permits unrestricted use, distribution, and reproduction in any medium, provided the original work is properly cited.

Aim. To evaluate the influence of interval between neoadjuvant chemoradiotherapy (NCRT) and surgery on oncological outcome. Methods. A systematic search was conducted in PubMed, the Cochrane Library, and Embase databases for publications reporting oncological outcomes of patients following rectal cancer surgery performed at different NCRT-surgery intervals. Relative risk (RR) of pathological complete response (pCR) among different intervals was pooled. Results. Fifteen retrospective cohort studies representing 4431 patients met the inclusion criteria. There was a significantly increased rate of pCR in patients treated with surgery followed 7 or 8 weeks later (RR, 1.45; 95\% CI, 1.18-1.78; and $P<0.01$ and RR, 1.49; 95\% CI, 1.15-1.92; and $P=0.002$, resp.). There is no consistent evidence of improved local control or overall survival with longer or shorter intervals. Conclusion. Performing surgery 7-8 weeks after the end of NCRT results in the highest chance of achieving pCR. For candidates of abdominoperineal resection before NCRT, these data support implementation of prolonging the interval after NCRT to optimize the chances of pCR and perhaps add to the possibility of ultimate organ preservation.

\section{Introduction}

The current standard of treatment for locally advanced rectal cancer, that is, stage cT3-4/Nx or cTx/N1-2 disease, consists of neoadjuvant chemoradiotherapy (NCRT), followed by radical total mesorectal excision (TME) (plus or minus further adjuvant chemotherapy) [1]. A recent published metaanalysis of randomized controlled trials suggested that preoperative chemoradiotherapy improves local control compared with surgery alone or surgery with neoadjuvant radiotherapy [2]. In addition, neoadjuvant therapy may result in complete eradication of all viable tumor cells from the primary tumor site as well as from the regional lymph nodes, representing pathological complete response (pCR) in this setting. Furthermore, complete response to preoperative NCRT is indicative of better long-term outcomes with low rates of local recurrence and distant failure [3].

A few small studies have recently investigated the influence of the length of the interval between NCRT and surgery
(NCRT-surgery interval) on morbidity, resectability, and tumor response $[4,5]$. However, the optimal NCRT-surgery interval that allows for maximal tumor regression is still unknown, and quantitative summarization of the supporting evidence of each time interval is not available. The purpose of this systematic review was, therefore, to examine the impact of the NCRT-surgery interval on the oncological outcome.

\section{Material and Methods}

2.1. Data Sources and Searches. We searched PubMed, the Cochrane Library, and Embase databases for relevant articles published until February 1, 2015; no lower date limit was applied. The search was limited initially to English publications. Electronic database searches were performed with the Boolean combination [(Interval OR time OR timing) AND rectal AND (carcinoma OR cancer) AND (Chemoradiotherapy OR Radiotherapy OR radiation OR neoadjuvant)] in all 
fields. In addition, the reference lists of included studies and related publications were screened for additional trials.

2.2. Study Selection. Two authors independently screened each unique record identified by the searches. Clinical trials meeting the following criteria were included in the metaanalysis: (i) the study directly compared pCR rates between patients in whom surgery for rectal cancer was performed at different intervals after NCRT. (ii) Patients were reported to undergo neoadjuvant radiation-based therapy and concurrent chemotherapy before surgery. (iii) The pCR rates were reported according to longer interval (NCRT-surgery interval longer than cutoff point) and shorter interval (NCRT-surgery interval shorter than cutoff point) categories. Studies that defined more than 2 time intervals were also accepted. (iv) The study had to report comparable data on pCR rates after NCRT of each time interval category. We excluded studies that were not published as full reports, such as conference abstracts and letters to editors. In case of multiple publications on the same study, the most recent information was used. Discrepancies in study selection were resolved by consensus.

2.3. Data Extraction and Study Assessment. To avoid bias in the data-abstraction process, two authors assessed the quality, extracted the data, and assessed the risk of bias independently. Any discrepancies between the authors were resolved by consensus. Details about the author, publication year, number of patients, treatment information, patient baseline characteristics, definitions of the pCR, pCR rates, and sphincter preservation rates of different groups and prognosis results were extracted from the included studies. When studies compared pCR rates between more than 2 time intervals, data were collected from each group separately. Methodological quality was assessed using Newcastle-Ottawa quality assessment scale.

2.4. Statistical Analysis. Rates of pCR was the primary endpoint, and local recurrence rates, distant metastases rates, overall survival rates, and sphincter preservation rates were the secondary endpoints. All statistical analysis was implemented with Review Manager version 5.3 software (Cochrane Collaboration, Denmark). To calculate relative risk (RR), patients of longer interval group were compared only with those of shorter interval in the same clinical trial. RR together with the $95 \%$ confidence interval (CI) was used as summary statistics for dichotomous data. We explored a relationship between pCR rates and time intervals by dividing patients into six categories based on NCRT-surgery intervals. We assessed statistical heterogeneity with $I^{2}$ statistics. Statistical heterogeneity between groups was considered relevant for comparisons with $I^{2}$ statistics of $>50 \%$. The $Z$-test for overall effect and its two-sided $P$ value were assessed. Significant difference was considered to be present for $P<0.05$.

2.5. Assessment of Bias Risk of the Included Studies and Sensitivity Analysis. Publication bias was not evaluated because of the small number of studies included in each subgroup.
Sensitivity analysis was performed for studies with same definition of pCR.

\section{Results}

Our search yielded a total of 3053 potentially relevant clinical studies, of which 2202 were among other themes. We then excluded review articles $(n=292)$, case reports $(n=135)$, comments $(n=21)$, letters $(n=18)$, and articles written in other languages $(n=351)$. In total, 34 articles were retrieved for full-text review. Of these, 6 articles were excluded due to lack of relevant data, 1 due to lack of a control arm, 7 due to failure to define a precise cutoff point of NCRTsurgery interval, and 5 due to failure to report postoperative pathologic outcome. The remaining 15 studies comprised a total of 4431 individuals, which constituted the material for the current review [6-20] (see Figure 1 for the selection process of these studies).

3.1. Characteristics of Studies Included in the Meta-Analysis. Fifteen retrospective cohort studies were included [6-20]. The studies included in this review are heterogeneous in the time intervals that they compared, ranging from $\leq 5$ weeks versus $>5$ weeks to $\leq 12$ weeks versus $>12$ weeks. All of the studies reported on patients who received NCRT. Although the exact nature of preoperative radiotherapy differed between the studies examined in this review, the majority of studies administered 45 to 50.4 Gy delivered over a period of 5 to 6 weeks. All of the studies reported on patients who received 5-fluorouracil- (FU-) based chemotherapy, though the chemotherapy regimens used varied. The common factors influencing NCRT-surgery intervals reported by most studies were surgeons' policy regarding the timing of operation, bed availability on the surgical wards, and comorbidities. The precise definition of complete pathological response was consistent among included studies, except Fang et al. [16] who defined pCR as "pT0 and any pN," and one patient who had a lymph node metastasis (ypT0N2) was therefore included in pCR group. Tulchinsky et al. [9] pooled $\mathrm{pCR}$ rates and near-pCR rates together for the analyses. The average methodological quality score was 6.7 (minimum 5, maximum 7) (Table 1).

The clinicopathologic data of the study populations are summarized in Table 2. No differences in average height of tumors from the anal verge were identified between shorter and longer intervals in any of the studies, except Sirohi et al. [20] who reported that height of tumors from the anal verge in shorter interval group was higher $(6 \mathrm{~cm}$ versus $4 \mathrm{~cm}$; $P=0.045)$. The majority of studies reported that neoadjuvant therapy was given to all patients with stage II or stage III rectal cancer, or with tumors that threatened circumferential resection margin. None of the included studies reported a significant difference in distribution of clinical T-stage and $\mathrm{N}$-stage between shorter and longer intervals cohorts.

3.2. Complete Pathological Response. Four studies reported a significantly increased rate of pCR after neoadjuvant therapy when patients were operated on at an interval $>7$ weeks 


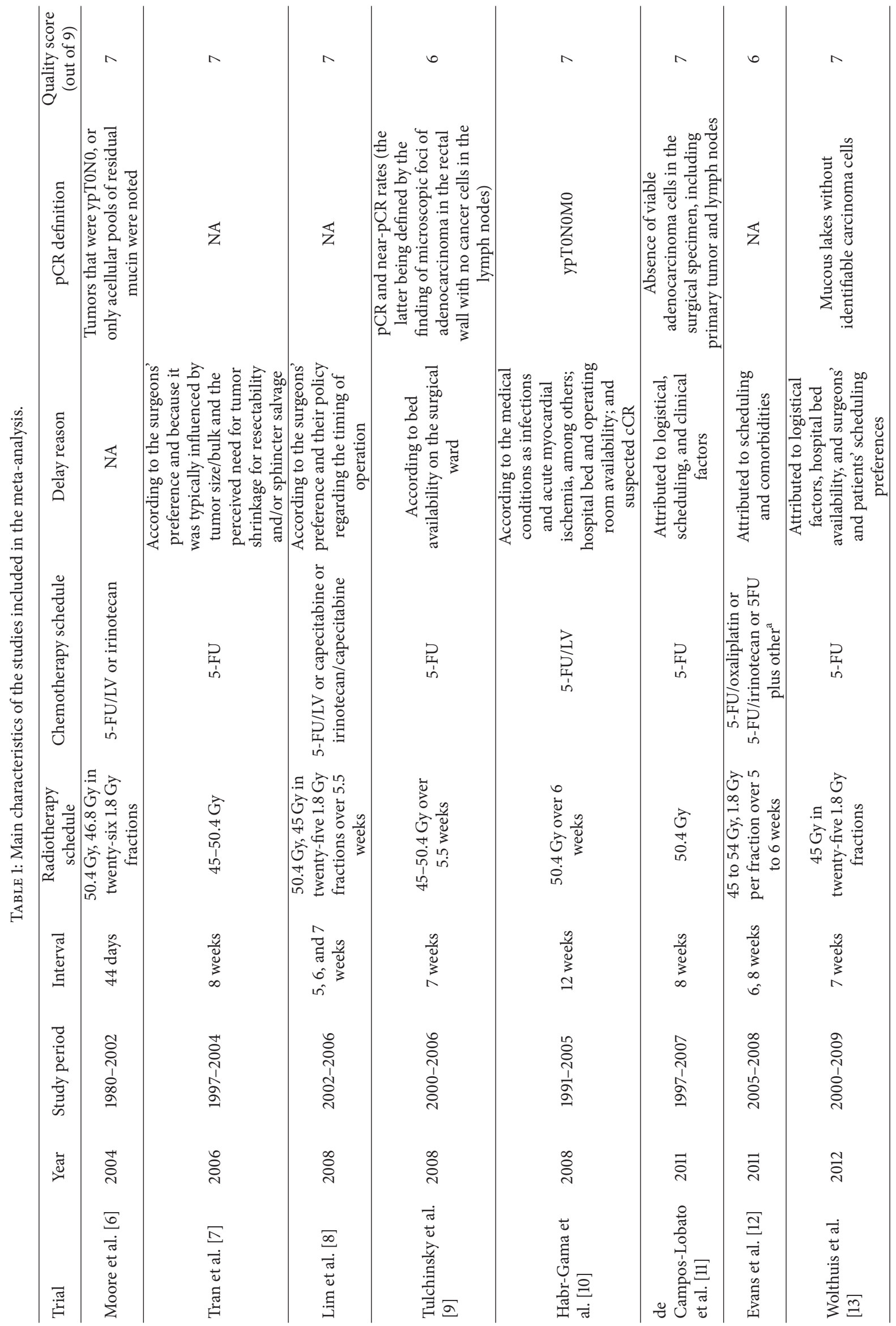




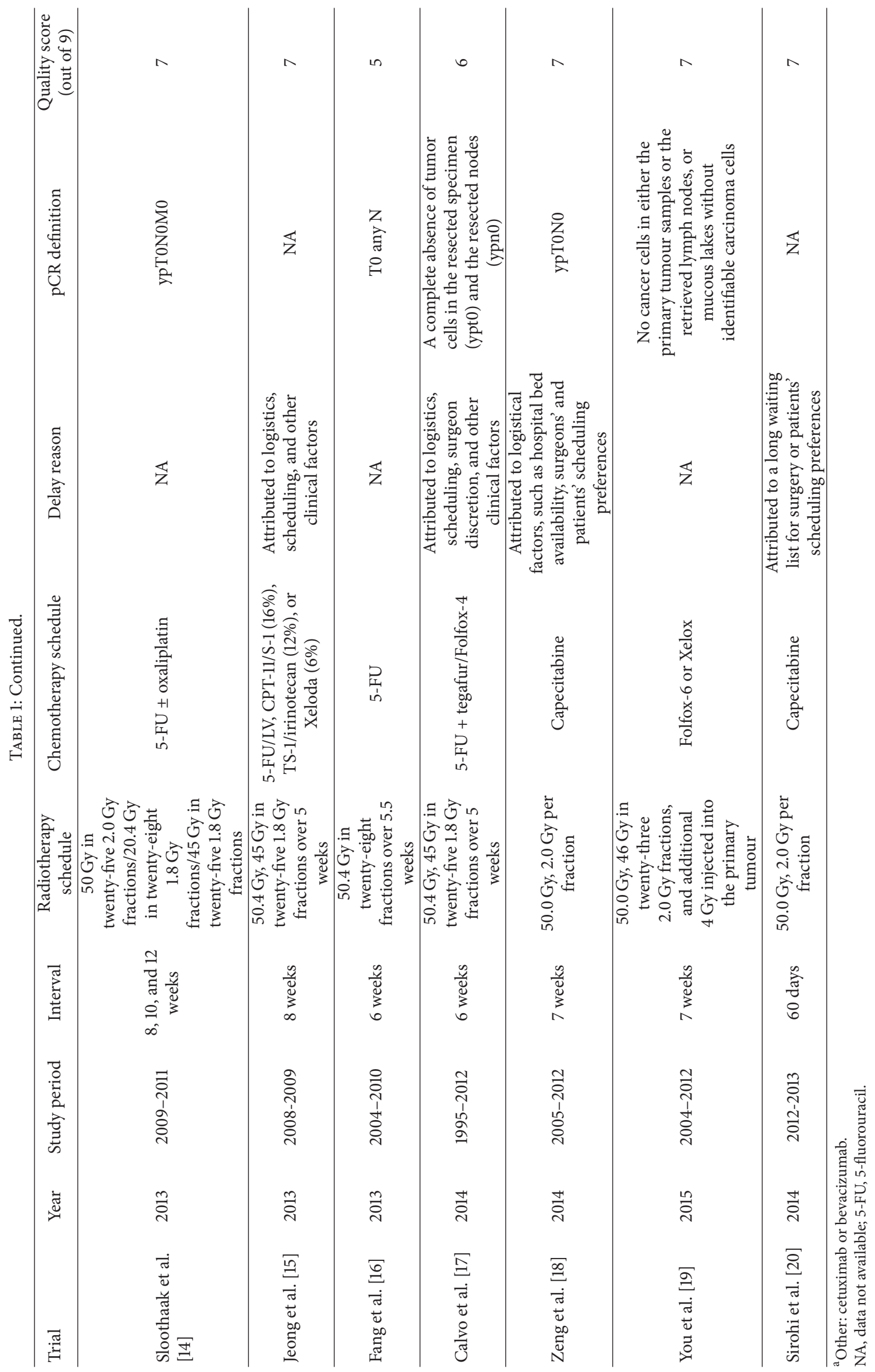




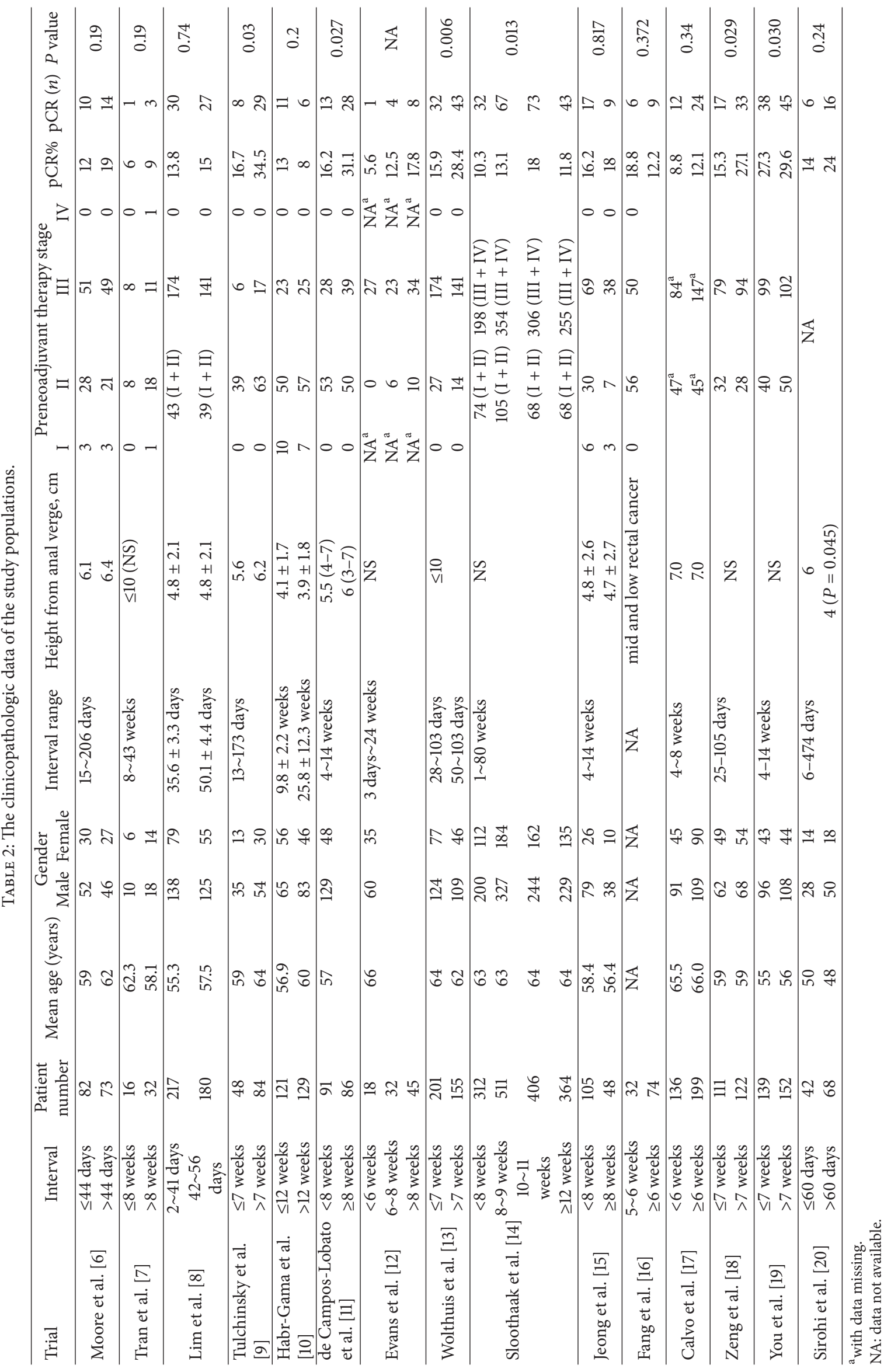




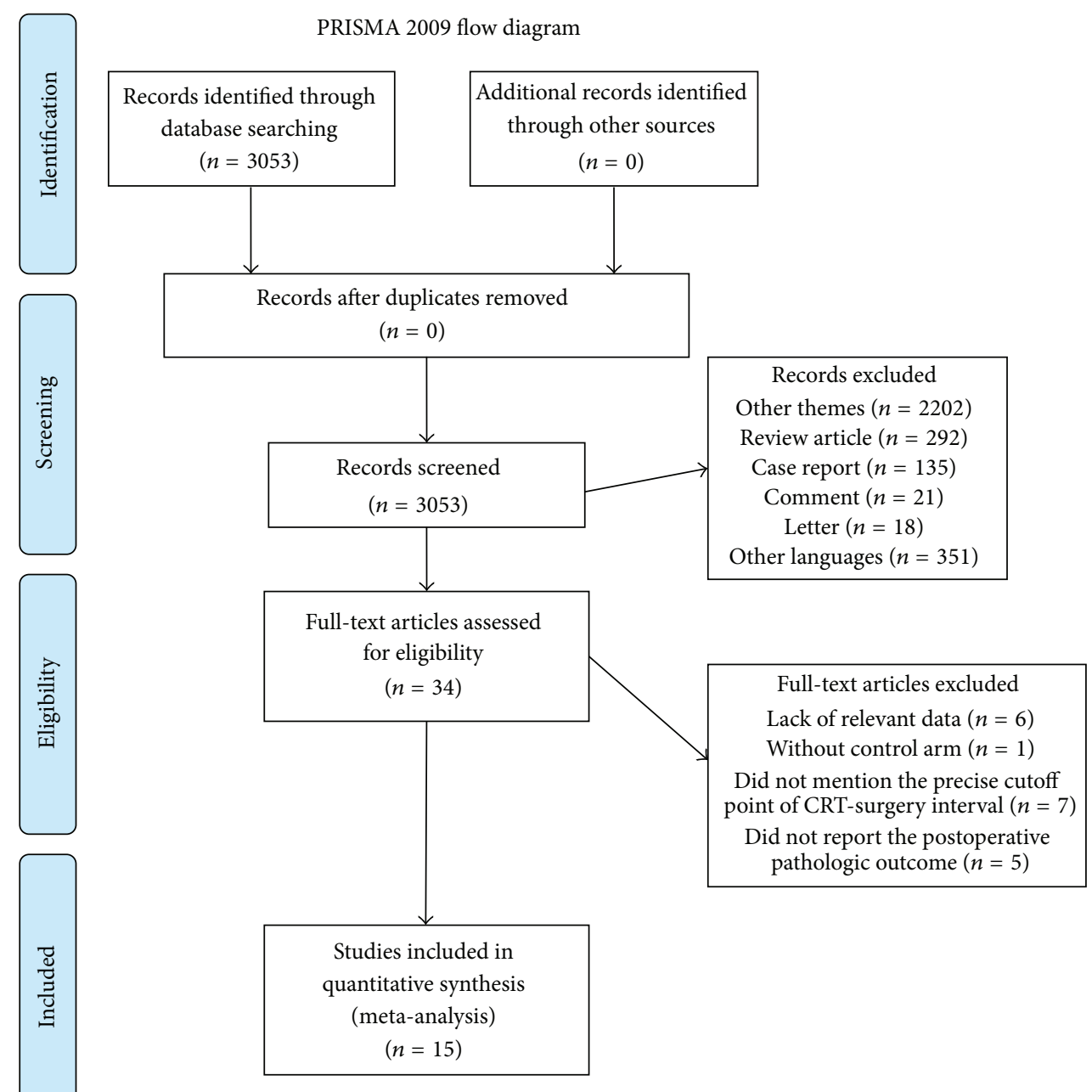

FIGURE 1: Flowchart of search process.

TABLE 3: Meta-analysis of pCR rate according to time intervals.

\begin{tabular}{|c|c|c|c|c|c|c|c|}
\hline \multirow{2}{*}{ Time intervals } & \multirow{2}{*}{ Number of studies } & \multirow{2}{*}{ Longer time intervals $(n) /$ shorter intervals $(n)$} & \multicolumn{2}{|c|}{ Test for heterogeneity } & \multirow{2}{*}{$\mathrm{RR}$} & \multirow{2}{*}{$95 \% \mathrm{CI}$} & \multirow{2}{*}{$P$ value } \\
\hline & & & $I^{2}$ & $P$ value & & & \\
\hline 5 weeks & 1 & $309 / 88$ & - & - & 0.67 & $0.40-1.12$ & 0.13 \\
\hline 6 weeks & 5 & $640 / 448$ & $28 \%$ & 0.23 & 1.03 & $0.76-1.42$ & 0.83 \\
\hline 7 weeks & 5 & $630 / 779$ & $25 \%$ & 0.26 & 1.45 & $1.18-1.78$ & $<0.01$ \\
\hline 8 weeks & 6 & $1568 / 608$ & $0 \%$ & 0.92 & 1.49 & $1.15-1.92$ & 0.002 \\
\hline 10 weeks & 1 & $770 / 543$ & - & - & 0.83 & $0.65-1.06$ & 0.13 \\
\hline 12 weeks & 2 & $493 / 1350$ & $0 \%$ & 0.41 & 0.81 & $0.60-1.08$ & 0.15 \\
\hline
\end{tabular}

CI, confidence interval.

$[9,13,18,19]$, and de Campos-Lobato et al. [11] found that a waiting interval of $\geq 8$ weeks was associated with a higher rate of $\mathrm{pCR}$. The highest $\mathrm{pCR}$ rates were observed in patients undergoing surgery on 10 to 11 weeks after the end of NCRT in the large series reported by Sloothaak et al. [14]. Seven studies reported an insignificant trend toward increased pCR rates in longer interval group $[6-8,12,15,17,20]$. No increase in rates of complete response was identified in 2 studies $[10,16]$.
3.3. Meta-Analysis of $p C R$ Rate. The reported $\mathrm{pCR}$ rates ranged from $8.3 \%$ to $28.0 \%$ [6-20]. In order to determine the particular contribution of time intervals of NCRT-surgery to the occurrence of $\mathrm{PCR}$, a meta-analysis was performed to calculate the RR associated with longer time intervals at beyond $5,6,7,8,10$, or 12 weeks when compared to shorter intervals (Table 3, Figures 2-5). No heterogeneity was found among these studies included in the analysis $\left(I^{2}<50 \%\right.$ each). Using 


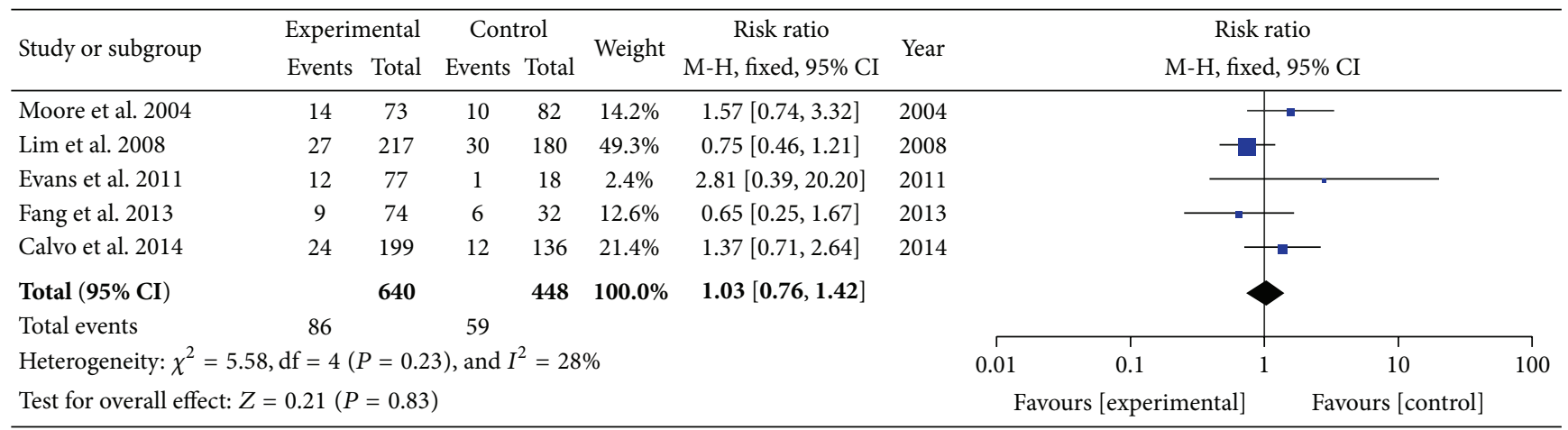

FIGURE 2: Standard forest plot of the RR for pCR rates comparing longer time intervals with shorter intervals at 6 weeks.

\begin{tabular}{|c|c|c|c|c|c|c|c|c|c|c|c|c|}
\hline \multirow{3}{*}{$\begin{array}{l}\text { Study or subgroup } \\
\text { Tulchinsky et al. } 2008\end{array}$} & \multicolumn{2}{|c|}{ Experimental } & \multirow{2}{*}{\multicolumn{2}{|c|}{$\begin{array}{c}\text { Control } \\
\text { Events Total }\end{array}$}} & \multirow{2}{*}{ Weight } & \multirow{2}{*}{$\begin{array}{c}\text { Risk ratio } \\
\text { M-H, fixed, 95\% CI }\end{array}$} & \multirow{2}{*}{ Year } & \multirow{2}{*}{\multicolumn{5}{|c|}{$\begin{array}{c}\text { Risk ratio } \\
\text { M-H, fixed, 95\% CI }\end{array}$}} \\
\hline & \multirow{2}{*}{$\begin{array}{c}\text { Events } \\
29\end{array}$} & \multirow{2}{*}{$\begin{array}{c}\text { Total } \\
84\end{array}$} & & & & & & & & & & \\
\hline & & & 8 & 48 & $8.6 \%$ & $2.07[1.03,4.16]$ & 2008 & & & &. & \\
\hline Lim et al. 2008 & 19 & 117 & 38 & 280 & $19.0 \%$ & $1.20[0.72,1.99]$ & 2008 & & & - & & \\
\hline Wolthuis et al. 2012 & 43 & 155 & 32 & 201 & $23.6 \%$ & $1.74[1.16,2.62]$ & 2012 & & & & - & \\
\hline Zeng et al. 2014 & 33 & 122 & 17 & 111 & $15.1 \%$ & $1.77[1.04,2.99]$ & 2014 & & & & - & \\
\hline You et al. 2015 & 45 & 152 & 38 & 139 & $33.7 \%$ & $1.08[0.75,1.56]$ & 2014 & & & & & \\
\hline Total $(95 \% \mathrm{CI})$ & & 630 & & 779 & $100.0 \%$ & $1.45[1.18,1.78]$ & & & & & & \\
\hline Total events & 169 & & 133 & & & & & & & & & \\
\hline Heterogeneity: $\chi^{2}=5$. & $\mathrm{df}=4$ & $(P=0$. & 26), and & $I^{2}=2$ & & & & 0.01 & 0.1 & 1 & 10 & 100 \\
\hline Test for overall effect: 2 & $=3.51(P$ & $P=0.0$ & & & & & & & {$[\operatorname{expe}$} & & Favours $[\mathrm{cc}$ & \\
\hline
\end{tabular}

FIGURE 3: Standard forest plot of the RR for pCR rates comparing longer time intervals with shorter intervals at 7 weeks.

\begin{tabular}{|c|c|c|c|c|c|c|c|c|c|c|c|c|}
\hline \multirow{3}{*}{$\begin{array}{l}\text { Study or subgroup } \\
\text { Tran et al. } 2006\end{array}$} & \multicolumn{2}{|c|}{ Experimental } & \multicolumn{2}{|c|}{ Control } & \multirow{2}{*}{ Weight } & \multirow{2}{*}{$\begin{array}{c}\text { Risk ratio } \\
\text { M-H, fixed, 95\% CI }\end{array}$} & \multirow{2}{*}{ Year } & \multirow{2}{*}{\multicolumn{5}{|c|}{$\begin{array}{c}\text { Risk ratio } \\
\text { M-H, fixed, 95\% CI }\end{array}$}} \\
\hline & \multirow{2}{*}{$\frac{\text { Events }}{3}$} & \multirow{2}{*}{$\begin{array}{c}\text { Total } \\
32\end{array}$} & \multirow{2}{*}{$\begin{array}{c}\text { Events } \\
1\end{array}$} & \multirow{2}{*}{$\begin{array}{c}\text { Total } \\
16\end{array}$} & & & & & & & & \\
\hline & & & & & \multirow{2}{*}{$\begin{array}{c}1.5 \% \\
15.4 \%\end{array}$} & \multirow{2}{*}{$\begin{array}{c}1.50[0.17,13.30] \\
1.90[1.06,3.42]\end{array}$} & 2006 & & & & & \\
\hline de Campos-Lobato et al. 2011 & 128 & 94 & 13 & 83 & & & 2011 & & & & -- & \\
\hline Evans et al. 2011 & 8 & 45 & 5 & 50 & $5.3 \%$ & $1.78[0.63,5.04]$ & 2011 & & & & & \\
\hline Sloothaak et al. 2013 & 183 & 1281 & 32 & 312 & $57.5 \%$ & $1.39[0.98,1.99]$ & 2013 & & & & & \\
\hline Jeong et al. 2013 & 9 & 48 & 17 & 105 & $11.9 \%$ & $1.16[0.56,2.41]$ & 2013 & & & & & \\
\hline Sirohi et al. 2014 & 16 & 68 & 6 & 42 & $8.3 \%$ & $1.65[0.70,3.88]$ & 2014 & & & & & \\
\hline Total $(95 \% \mathrm{CI})$ & & 1568 & & 608 & $100.0 \%$ & $1.49[1.15,1.92]$ & & & & & & \\
\hline Total events & 247 & & 74 & & & & & & & & & \\
\hline Heterogeneity: $\chi^{2}=1.42, \mathrm{df}=$ & $=5(P=$ & $=0.92)$, & and $I^{2}=$ & $=0 \%$ & & & & 0.01 & 0.1 & & 10 & 100 \\
\hline Test for overall effect: $Z=3.0$ & $03(P=$ & $0.002)$ & & & & & & Fav & exper & & Favours $[\mathrm{co}$ & \\
\hline
\end{tabular}

FIGURE 4: Standard forest plot of the RR for pCR rates comparing longer time intervals with shorter intervals at 8 weeks.

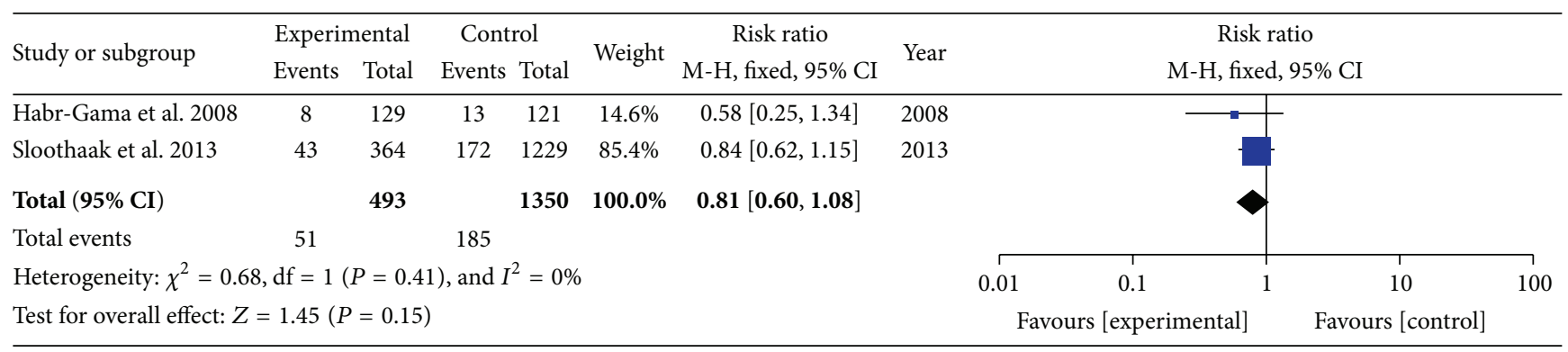

FIGURE 5: Standard forest plot of the RR for pCR rates comparing longer time intervals with shorter intervals at 12 weeks. 


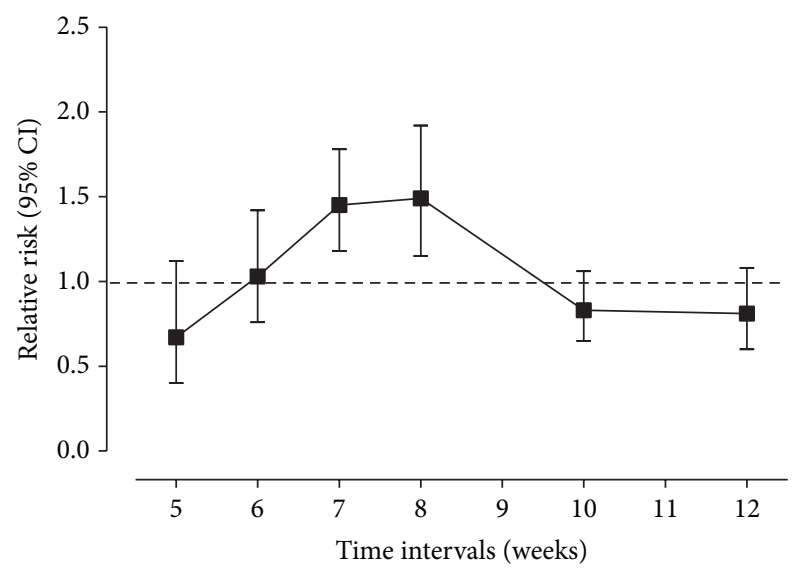

FIGURE 6: The relationship between time intervals and RR of pCR rates. The center of each black square is placed at the summary point estimate, and each vertical line shows the $95 \%$ confidence interval about the summary estimate.

a fixed-effect model, there was a significantly increased rate of pCR in patients treated with surgery followed 7 or 8 weeks later (RR, 1.45; 95\% CI, 1.18-1.78; and $P<0.01$ and RR, 1.49; 95\% CI, 1.15-1.92; and $P=0.002)$. No significant differences were found between shorter and longer intervals cohorts with respect to rate of pCR in earlier cutoff points of 5 and 6 weeks (RR, 0.67; 95\% CI, 0.40-1.12; and $P=0.13$ and RR, 1.03; 95\% $\mathrm{CI}, 0.76-1.42$; and $P=0.83$ ), and further extension beyond 10 or 12 weeks did not offer further advantages in increasing pCR rates (RR, 0.83; 95\% CI, 0.65-1.06; and $P=0.13$ and RR, 0.81 ; $95 \% \mathrm{CI}, 0.60-1.08$; and $P=0.15$ ). Sensitivity analyses excluding data from Tulchinsky et al. [9] to control for patient with near-pCR did not alter the results substantially (RR, 1.39; 95\% CI, 1.12 to 1.73 ; and $P=0.003$ ). Data from Fang et al. [16] was also excluded for sensitivity analyses because of different definition of pCR, and result showed no change (RR, 1.09; $95 \%$ CI, 0.78 to 1.52 ; and $P=0.61$ ).

We then plotted the pooled RR and 95\% CI of pCR rates of different intervals in a line chart. The highest summary point estimate in the RR of $\mathrm{pCR}$ rates was observed in patients receiving surgery beyond 8 weeks after the end of NCRT, which was associated with an approximately 49\% higher chance of achieving pCR than patients who were operated on less than 8 weeks after the end of NCRT. The corresponding figure for $\geq 7$ weeks' cutoff point was $45 \%$ (Figure 6).

3.4. Sphincter Preservation. Thirteen of the included studies reported rate of sphincter preservation (Table 4). None of the included studies reported a significant increase in rates of sphincter preservation with a longer interval. Habr-Gama et al. [10] reported a lower rate of sphincter preservation among patients undergoing surgery after a longer interval of $>12$ weeks, as patients in this study with a suspected cCR after NCRT were enrolled in a watch-and-wait protocol and were not managed by surgery until recurrence occurred.

3.5. Long-Term Outcome. Twelve of the included studies reported long-term outcomes (Table 4). Six of these found no significant difference in local recurrence rates, or local control rate, and distant metastases rate between patients who were operated on after shorter or longer intervals. A significantly higher rate of local recurrence was reported in patients undergoing surgery after a shorter interval $(<7,8$ weeks) reported by de Campos-Lobato et al. [11] and Zeng et al. [18] (10.5\% versus $1.2 \%, P=0.04$, and $12.9 \%$ versus $4.8 \%, P=0.025$, resp.). Tulchinsky et al. [9] reported a significant increase in distant metastases rate when surgery was performed after a shorter interval $<7$ weeks $(19 \%$ versus $6 \%, P=0.02)$. A significantly higher 5-year free-fromrecurrence rate was reported in patients undergoing surgery after an interval of $>8$ weeks reported by Wolthuis et al. [13] (73\% versus $83 \%, P=0.026$ ). In terms of overall survival, six studies found no significant difference between patients who were operated on after shorter or longer intervals except 1 study, in which Calvo et al. [17] identified a significant association between increased 5-year overall survival with prolonged interval of $\geq 6$ weeks.

\section{Discussion}

The optimal timing for surgery after neoadjuvant treatment for rectal cancer remains at large. The justification for an effort to examine optimal timing of surgery after preoperative radiation therapy stems from the Lyon R90-01 study [21], the only randomized trial to date that examines the time interval to surgery, in which outcomes after short (less than 2 weeks) and long (6-8 weeks) intervals following preoperative radiotherapy were compared. The longer interval was correlated with a significantly higher proportion of pathologic downstaging. Furthermore, this trial was the only one that demonstrated an increase of sphincter preservation following longer interval to surgery. Therefore, the 6-8-week interval between NCRT and surgery has become routine practice for rectal cancer. However, patients in this study received a currently unusual radiation dose (39 Gy in 13 fractions), did not routinely undergo TME, and did not receive preoperative chemotherapy. Furthermore, higher safe dose of radiation therapy (3 Gy per fraction) might impact the rate of sphincter preservation. Another ongoing multicentric randomized controlled trial (the GRECCAR6 study) only compares 7 and 11 weeks of delay between the end of NRCT and surgery of rectal cancer [22]. There is no previous studies evaluated the association between each time interval and rate of $\mathrm{pCR}$.

Tumor regression and radiation-induced necrosis are a time-dependent phenomenon [23]. The effects of chemoradiotherapy are based on the cell cycle, and oftentimes multiple cycles administered over the course of several months are necessary before effects are seen [24]. Therefore, there is enthusiasm for prolonging the currently accepted interval of 6-8 weeks in order to maximize the downstaging effect of NCRT and subsequently increase the pCR rate [25]. But will a longer interval even result in a superior rate of pCR? Dolinsky et al. [26] reported the rates of primary tumor downstaging were $42 \%, 58 \%$, and $71 \%$ for patients with intervals of $<6$, $6-8$, and $>8$ weeks, respectively, but an increase in the time interval did not affect the likelihood of achieving a pCR (OR $0.97,95 \% \mathrm{CI}, 0.78-1.21$, and $P=0.8$ ) in multivariate analysis. 


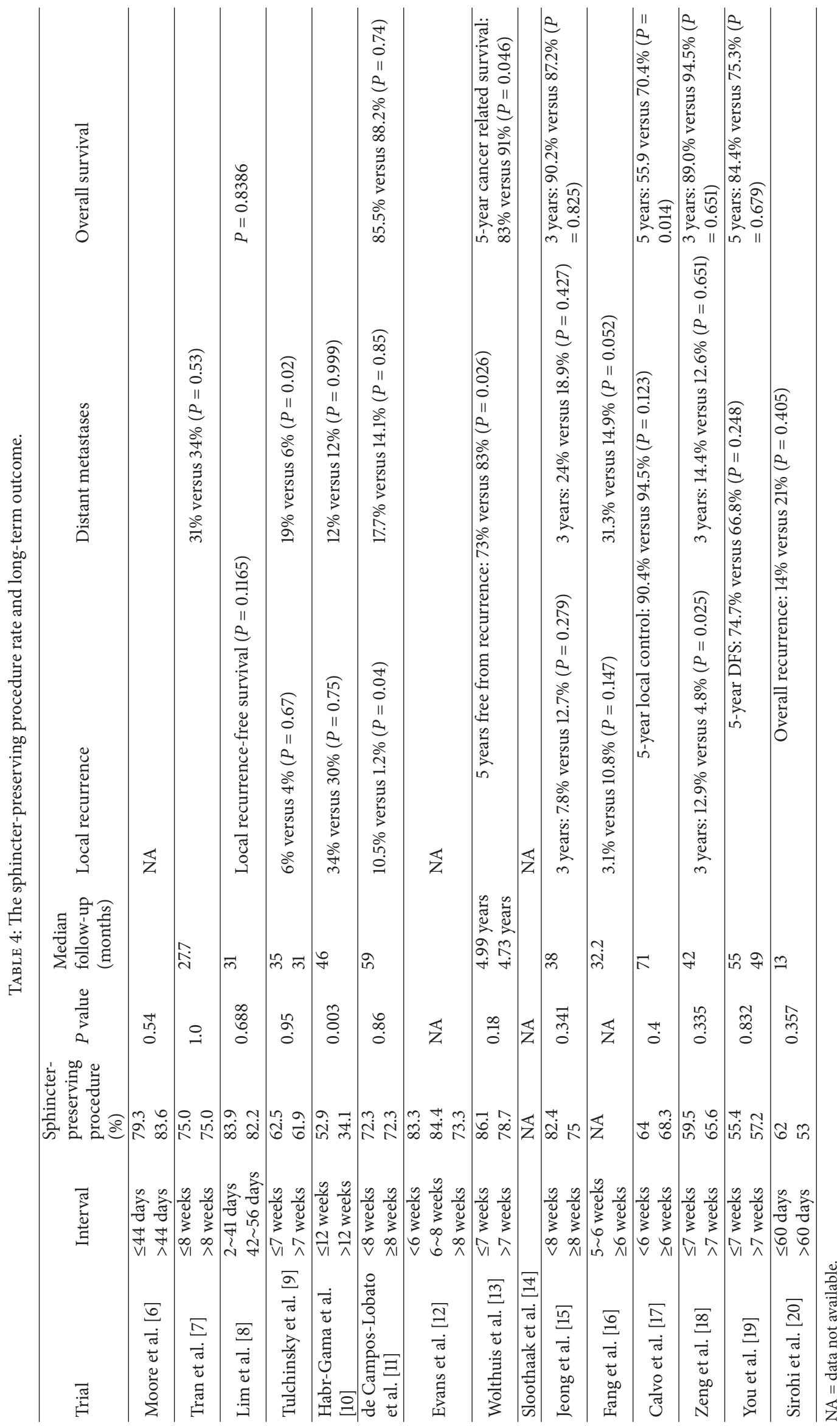


For short-course radiotherapy, there are 4 phase-III studies in the literature with randomized intervals. Two of these studies compared short-course radiotherapy and immediate surgery with short-course radiotherapy and delayed surgery and found that the ypCR rate was about $10 \%$ higher in the delayed-surgery group $[27,28]$. The comparison of shortcourse radiotherapy and delayed surgery with long-course chemoradiation in another randomized study revealed that the $\mathrm{pCR}$ rate was higher in the long-course chemoradiation groups [29]. An interim analysis of a multicentre randomized study that compared short-course radiotherapy and consolidation chemotherapy with long-course chemoradiation revealed a higher $\mathrm{pCR}$ rate in the short-course radiotherapy group [30].

A published meta-analysis demonstrated that a longer waiting interval (more than 6-8 weeks) from the end of NCRT increases the rate of pCR by $6 \%(\mathrm{RR}=1.42,95 \% \mathrm{CI}$, 1.19-1.68, and $P<0.0001$ ), with similar long-term outcomes and complication rates [31]. However, they failed to perform subgroup analysis according to each particular time point. As there was lack of consistency in the time intervals examined by the included studies, heterogeneity may well exist when data of all time intervals were pooled together. After including more trials (including 4 new published trials with 969 patients), we pooled data from different time intervals to perform a subgroup analysis. The present data demonstrated that delaying surgery until the seventh or eighth week after NCRT significantly increased rate of pCR in patients with rectal cancer (increasing rate by $49 \%$ and $45 \%$, resp.). Notably, no significant improvement of pCR rate was found when patients had operation beyond commonly accepted 6 weeks, as 6-week point is probably insufficient to reveal relevant differences. Thus, we establish the optimal window between 7-8 weeks to intervene surgically within the established 6-8 weeks' window.

There is, however, less data published regarding the results of increasing the interval prior to surgical intervention ( $>10$ weeks). Our data suggests that no significant difference in pCR rate was found when patients had a waiting interval of $\geq 10$ or 12 weeks. However, a potential limitation in our meta-analysis is that only two studies were included in these longer interval subgroups. Garcia-Aguilar et al. [23] indicated that patients operated on 11 13 weeks after NCRT had a pCR rate of $25 \%$, compared to $18 \%$ for patients operated on $6 \sim 8$ weeks after NCRT $(P=0.022)$. However, Stein et al. [24] did not find increased rate of pCR when surgery was performed at 10 to 14 weeks (14\%) in comparison with surgery at 4 to 8 weeks $(21 \%)$ after NCRT. In a recent published exploratory phase-2 trial, Garcia-Aguilar et al. [32] showed that adding up to six cycles of mFOLFOX6 chemotherapy between NCRT and surgery, meanwhile, delaying surgery after NCRT, increased the pCR rate. The increased number of cycles was significantly associated with an increased proportion of patients who achieved a pCR. The patients assigned to receive six cycles of mFOLFOX6 after chemoradiation with surgery 19 weeks after NCRT achieved a highest rate of pCR of $38 \%$, which is one of the highest proportions reported so far for stages II-III rectal cancer. However, the excellent clinical response was probably not due to longer interval but preoperative chemotherapy. In addition, a few issues need to be addressed. Waiting longer clearly benefits those patients that achieve a pCR, but over $20 \%$ do not respond to preoperative therapy and, in fact, the primary tumor continues to grow [33]. Another concern of delaying surgery is that longer intervals after preoperative radiation may increase the risk of emergence of distant subclinical tumor, which can grow to a metastasis-yielding volume and lead to the development of distant metastases [34]. Despite several studies reporting promising use of imaging technology to help in monitoring disease response during preoperative treatment, no robust imaging technology has been established for widespread clinical use $[35,36]$. Based on these concerns, further delaying time interval ( $>10$ weeks) may be inappropriate.

Tumor response to NCRT has been shown to be a predictor of less propensity for local or distant recurrence and improved survival [16]. Indications of tumor regression include downstaging, downsizing, complete, or nearly complete response. As the definitions of downstaging and downsizing varied widely among studies [37], complete pathological response to neoadjuvant therapy has become a widely measured endpoint in rectal cancer clinical trials [38]. In this study, we chose to define pCR as absence of viable adenocarcinoma cells in the surgical specimen, including primary tumor and lymph nodes. There is marked heterogeneity in reported pCR rates across included studies (8.3\%-28.0\%), probably due to different neoadjuvant therapy protocols and patients with different stages enrolled in. The sensitivity analyses were performed excluding the study in which patients with pCR $(n=26)$ and near-pCR $(n=11)$ were included [9], and another study in which one patient with pT0N2 stage was included in pCR group [16]. Results of sensitivity analyses suggest our findings were robust.

NCRT was reported to achieve tumor downsizing and downstaging, which might cause an increase in the tumor distance from the anal verge, meanwhile increasing the likelihood of sphincter preservation [39]. However, none of the studies included in this review reported a significant increase in rates of sphincter preservation with a longer interval. Although the longer interval to surgery was associated with a higher pCR rates in 4 phase-III trials regarding shortcourse radiotherapy, the sphincter preservation rate and long-term outcome were similar [27-29, 32]. When comparing with postoperative chemoradiotherapy, the benefit of sphincter preservation and survival was not confirmed for preoperative chemoradiotherapy by results of the German CAO/ARO/AIO-94 randomized phase-III trial $[40,41]$. In the phase-II trial by Garcia-Aguilar et al., waiting longer than 19 weeks was not associated with an increase in the proportion of patients who had a sphincter-saving procedure. However, patients with good response who refused to undergo TME but local excision were excluded from analyses [32]. Though longer interval was not demonstrated to increase in conventional sphincter-saving procedure (e.g., anterior resection), it can increase the rate of $\mathrm{pCR}$, meanwhile, increasing the proportion of organ preservation (e.g., local excision [42] or watch-and-wait policy [43, 44]) for patients with clinical complete response. 
Whether there is an association between time interval and prognosis remains controversial. The meta-analysis performed by Petrelli et al. reported similar long-term outcomes between longer and shorter interval groups, but this metaanalysis redichotomised all of the patients into two groups according to the 6-8 weeks' interval [31]. In the current review, local control, distant metastasis, and overall survival were reported to be similar between longer and shorter interval in most of included studies, though a few studies reported an association between longer intervals with better long-term outcome. We failed to perform a meta-analysis of survival data of each time interval group to compare prognosis of all of the possible intervals, as data were not available. Further studies investigating this are warranted.

Our study has the following limitations. First, all data were extracted from retrospective studies, but the majority of included studies reported patients with similar baseline demographic and oncologic characteristics in both groups. Tulchinsky et al. [9] reported that patients operated on at an interval $>7$ weeks were older at operation $(P=0.007)$, but they were able to show that age at operation was not a predictor for $\mathrm{pCR}$ and near-pCR $(P=0.57)$. Moreover, while most analyses were conducted from a retrospective perspective, the data used were collected prospectively in the highly standardized manner; neoadjuvant therapy schedule and our primary outcome of interest, pCR, are all routinely collected and objectively measured, thereby minimizing the problems of missing data and reporting bias. Second, the time intervals after NCRT showed significant heterogeneity among the included studies. As surgeons' policy regarding the timing of operation was reported to be the most common factor influencing NCRT-surgery intervals by most studies, various potential confounders could have been involved in the choice of interval times for the cases considered. For instance, patients with progressive or stable disease after NCRT might have surgery without further delay after completing NCRT, which caused selection bias. Third, we failed to perform a meta-analysis of survival data of each time interval group. Although time intervals of 7-8 weeks significantly increase chances of achieving pCR in present study, it is unclear whether this translates into long-term clinical benefit.

\section{Conclusion}

The results of this review demonstrate that performing surgery 7-8 weeks after the end of NCRT significantly increases rates of pCR. Increasing the interval prior to surgical intervention alone has no impact on long-term survival. For candidates of abdominoperineal resection before NCRT, these data support implementation of prolonging interval after NCRT to optimize the chances of $\mathrm{PCR}$ and perhaps add to the possibility of ultimate organ preservation. This is best addressed in the context of a randomized control trial.

\section{Abbreviations}

NCRT: Neoadjuvant chemoradiotherapy

pCR: Pathological complete response
RR: Relative risk

CIs: Confidence intervals.

\section{Conflict of Interests}

The authors have no conflict of interests to declare.

\section{Acknowledgment}

This work was financially supported by National Clinical Key Specialty Construction Project (General Surgery) of China.

\section{References}

[1] J. R. T. Monson, M. R. Weiser, W. D. Buie, G. J. Chang, and J. F. Rafferty, "Practice parameters for the management of rectal cancer (revised)," Diseases of the Colon and Rectum, vol. 56, no. 5, pp. 535-550, 2013.

[2] N. N. Rahbari, H. Elbers, V. Askoxylakis et al., "Neoadjuvant radiotherapy for rectal cancer: meta-analysis of randomized controlled trials," Annals of Surgical Oncology, vol. 20, no. 13, pp. 4169-4182, 2013.

[3] S. T. Martin, H. M. Heneghan, and D. C. Winter, "Systematic review and meta-analysis of outcomes following pathological complete response to neoadjuvant chemoradiotherapy for rectal cancer," The British Journal of Surgery, vol. 99, no. 7, pp. 918928, 2012.

[4] M. R. Weiser, H.-M. Quah, J. Shia et al., "Sphincter preservation in low rectal cancer is facilitated by preoperative chemoradiation and intersphincteric dissection," Annals of Surgery, vol. 249, no. 2, pp. 236-242, 2009.

[5] L.-J. Kuo, M.-C. Liu, J. J.-M. Jian et al., "Is final TNM staging a predictor for survival in locally advanced rectal cancer after preoperative chemoradiation therapy?" Annals of Surgical Oncology, vol. 14, no. 10, pp. 2766-2772, 2007.

[6] H. G. Moore, A. E. Gittleman, B. D. Minsky et al., "Rate of pathologic complete response with increased interval between preoperative combined modality therapy and rectal cancer resection," Diseases of the Colon and Rectum, vol. 47, no. 3, pp. 279-286, 2004.

[7] C.-L. Tran, S. Udani, A. Holt, T. Arnell, R. Kumar, and M. J. Stamos, "Evaluation of safety of increased time interval between chemoradiation and resection for rectal cancer," The American Journal of Surgery, vol. 192, no. 6, pp. 873-877, 2006.

[8] S.-B. Lim, H. S. Choi, S.-Y. Jeong et al., "Optimal surgery time after preoperative chemoradiotherapy for locally advanced rectal cancers," Annals of Surgery, vol. 248, no. 2, pp. 243-251, 2008.

[9] H. Tulchinsky, E. Shmueli, A. Figer, J. M. Klausner, and M. Rabau, "An interval $>7$ weeks between neoadjuvant therapy and surgery improves pathologic complete response and diseasefree survival in patients with locally advanced rectal cancer," Annals of Surgical Oncology, vol. 15, no. 10, pp. 2661-2667, 2008.

[10] A. Habr-Gama, R. O. Perez, I. Proscurshim et al., "Interval between surgery and neoadjuvant chemoradiation therapy for distal rectal cancer: does delayed surgery have an impact on outcome?" International Journal of Radiation Oncology, Biology, Physics, vol. 71, no. 4, pp. 1181-1188, 2008.

[11] L. F. de Campos-Lobato, D. P. Geisler, A. D. L. Moreira, L. Stocchi, D. Dietz, and M. F. Kalady, "Neoadjuvant therapy for rectal cancer: the impact of longer interval between chemoradiation 
and surgery," Journal of Gastrointestinal Surgery, vol. 15, no. 3, pp. 444-450, 2011.

[12] J. Evans, D. Tait, I. Swift et al., "Timing of surgery following preoperative therapy in rectal cancer: the need for a prospective randomized trial?" Diseases of the Colon and Rectum, vol. 54, no. 10, pp. 1251-1259, 2011.

[13] A. M. Wolthuis, F. Penninckx, K. Haustermans et al., "Impact of interval between neoadjuvant chemoradiotherapy and TME for locally advanced rectal cancer on pathologic response and oncologic outcome," Annals of Surgical Oncology, vol. 19, no. 9, pp. 2833-2841, 2012.

[14] D. A. Sloothaak, D. E. Geijsen, N. J. van Leersum et al., "Optimal time interval between neoadjuvant chemoradiotherapy and surgery for rectal cancer," British Journal of Surgery, vol. 100, no. 7, pp. 933-939, 2013.

[15] D. H. Jeong, H. B. Lee, H. Hur, B. S. Min, S. H. Baik, and N. K. Kim, "Optimal timing of surgery after neoadjuvant chemoradiation therapy in locally advanced rectal cancer," Journal of the Korean Surgical Society, vol. 84, no. 6, pp. 338345, 2013.

[16] C. B. Fang, C. M. C. D. N. Gomes, F. B. Formiga, V. A. Fonseca, M. P. Carvalho, and W. A. Klug, "Is the delayed surgery after neoadjuvant chemoradiation beneficial for locally advanced rectal cancer?" ABCD Arquivos Brasileiros de Cirurgia Digestiva (São Paulo), vol. 26, no. 1, pp. 31-35, 2013.

[17] F. A. Calvo, V. Morillo, M. Santos et al., "Interval between neoadjuvant treatment and definitive surgery in locally advanced rectal cancer: impact on response and oncologic outcomes," Journal of Cancer Research and Clinical Oncology, vol. 140, no. 10, pp. 1651-1660, 2014.

[18] W.-G. Zeng, Z.-X. Zhou, J.-W. Liang et al., "Impact of interval between neoadjuvant chemoradiotherapy and surgery for rectal cancer on surgical and oncologic outcome," Journal of Surgical Oncology, vol. 110, no. 4, pp. 463-467, 2014.

[19] K.-Y. You, R. Huang, L.-N. Zhang et al., "Tailored selection of the interval between neoadjuvant chemoradiotherapy and surgery for locally advanced rectal cancer: analysis based on the pathologic stage or chemoradiation response," Journal of Cancer Research and Clinical Oncology, vol. 141, no. 4, pp. 719-728, 2015.

[20] B. Sirohi, S. G. Barreto, S. Patkar et al., "Down-staging following neoadjuvant chemo-radiotherapy for locally advanced rectal cancer: does timing of surgery really matter?" Indian Journal of Medical and Paediatric Oncology, vol. 35, no. 4, pp. 263-266, 2014.

[21] Y. Francois, C. J. Nemoz, J. Baulieux et al., "Influence of the interval between preoperative radiation therapy and surgery on downstaging and on the rate of sphincter-sparing surgery for rectal cancer: the Lyon R90-01 randomized trial," Journal of Clinical Oncology, vol. 17, no. 8, pp. 2396-2402, 1999.

[22] J. H. Lefevre, A. Rousseau, M. Svrcek, Y. Parc, T. Simon, and E. Tiret, "A multicentric randomized controlled trial on the impact of lengthening the interval between neoadjuvant radiochemotherapy and surgery on complete pathological response in rectal cancer (GRECCAR- 6 trial): rationale and design," BMC Cancer, vol. 13, article 417, 2013.

[23] J. Garcia-Aguilar, D. D. Smith, K. Avila et al., "Optimal timing of surgery after chemoradiation for advanced rectal cancer: preliminary results of a multicenter, nonrandomized phase II prospective trial," Annals of Surgery, vol. 254, no. 1, pp. 97-102, 2011.

[24] D. E. Stein, N. N. Mahmoud, P. R. Anné et al., "Longer time interval between completion of neoadjuvant chemoradiation and surgical resection does not improve downstaging of rectal carcinoma," Diseases of the Colon and Rectum, vol. 46, no. 4, pp. 448-453, 2003.

[25] D. Harris, "Optimal time interval between neoadjuvant chemoradiotherapy and surgery for rectal cancer (Br J Surg 2013; 100: 933-939)," British Journal of Surgery, vol. 100, no. 7, pp. 939-940, 2013.

[26] C. Dolinsky, N. Mahmoud, R. Mick et al., "Effect of time interval between surgery and preoperative chemoradiotherapy with 5fluorouracil (5-FU) or 5-FU/oxaliplatin (5FU/OX) for rectal cancer," International Journal of Radiation Oncology, Biology, Physics, vol. 63, article S277, 2005.

[27] D. Pettersson, E. Lörinc, T. Holm et al., "Tumour regression in the randomized Stockholm III Trial of radiotherapy regimens for rectal cancer," British Journal of Surgery, vol. 102, no. 8, pp. 972-978, 2015.

[28] R. Pach, J. Kulig, P. Richter, T. Gach, M. Szura, and T. Kowalska, "Randomized clinical trial on preoperative radiotherapy 25 Gy in rectal cancer-treatment results at 5-year follow-up," Langenbeck's Archives of Surgery, vol. 397, no. 5, pp. 801-807, 2012.

[29] T. Latkauskas, H. Pauzas, I. Gineikiene et al., "Initial results of a randomized controlled trial comparing clinical and pathological downstaging of rectal cancer after preoperative shortcourse radiotherapy or long-term chemoradiotherapy, both with delayed surgery," Colorectal Disease, vol. 14, no. 3, pp. 294298, 2012.

[30] K. Bujko, A. Nasierowska-Guttmejer, L. Wyrwicz et al., "Neoadjuvant treatment for unresectable rectal cancer: an interim analysis of a multicentre randomized study, Radiotherapy \& Oncology, vol. 107, no. 2, pp. 171-177, 2013.

[31] F. Petrelli, G. Sgroi, E. Sarti, and S. Barni, "Increasing the interval between neoadjuvant chemoradiotherapy and surgery in rectal cancer: a meta-analysis of published studies," Annals of Surgery, 2015.

[32] J. Garcia-Aguilar, O. S. Chow, D. D. Smith et al., "Effect of adding mFOLFOX6 after neoadjuvant chemoradiation in locally advanced rectal cancer: a multicentre, phase 2 trial," The Lancet Oncology, vol. 16, no. 8, pp. 957-966, 2015.

[33] S. Huerta, "Interval between neoadjuvant chemoradiation and surgery for the management of rectal cancer," Journal of Gastrointestinal Surgery, vol. 15, no. 7, p. 1292, 2011.

[34] H. R. Withers and K. Haustermans, "Where next with preoperative radiation therapy for rectal cancer?" International Journal of Radiation Oncology Biology Physics, vol. 58, no. 2, pp. 597-602, 2004.

[35] R. W. Motson, J. S. Khan, T. H. A. Arulampalam, R. C. T. Austin, N. Lacey, and B. Sizer, "Laparoscopic total mesorectal excision following long course chemoradiotherapy for locally advanced rectal cancer," Surgical Endoscopy, vol. 25, no. 6, pp. 1753-1760, 2011.

[36] C. Hottenrott, "Timing of laparoscopic surgery in the neoadjuvant treatment of rectal cancer," Surgical Endoscopy, vol. 26, no. 3, pp. 884-885, 2012.

[37] J. D. Foster, E. L. Jones, S. Falk, E. J. Cooper, and N. K. Francis, "Timing of surgery after long-course neoadjuvant chemoradiotherapy for rectal cancer: a systematic review of the literature," Diseases of the Colon and Rectum, vol. 56, no. 7, pp. 921-930, 2013.

[38] M. Maas, P. J. Nelemans, V. Valentini et al., "Long-term outcome in patients with a pathological complete response 
after chemoradiation for rectal cancer: a pooled analysis of individual patient data," The Lancet Oncology, vol. 11, no. 9, pp. 835-844, 2010.

[39] M. H. Ihn, Y. H. Kim, D.-W. Kim et al., "Effects of preoperative chemoradiotherapy on the likelihood of sphincter preservation surgery in locally advanced distal rectal cancer: a longitudinal study based on pelvic magnetic resonance imaging," Annals of Surgical Oncology, vol. 22, no. 7, pp. 2159-2167, 2015.

[40] R. Sauer, H. Becker, W. Hohenberger et al., "Preoperative versus postoperative chemoradiotherapy for rectal cancer," The New England Journal of Medicine, vol. 351, no. 17, pp. 1731-1740, 2004.

[41] R. Sauer, T. Liersch, S. Merkel et al., "Preoperative versus postoperative chemoradiotherapy for locally advanced rectal cancer: results of the German CAO/ARO/AIO-94 randomized phase III trial after a median follow-up of 11 years," Journal of Clinical Oncology, vol. 30, no. 16, pp. 1926-1933, 2012.

[42] C. Pericay, X. Serra-Aracil, J. Ocaña-Rojas et al., "Further evidence for preoperative chemoradiotherapy and transanal endoscopic surgery (TEM) in T2-3s,N0,M0 rectal cancer," Clinical \& Translational Oncology, 2015.

[43] M. Rupinski, M. Szczepkowski, M. Malinowska et al., "Watch and wait policy after preoperative radiotherapy for rectal cancer; Management of residual lesions that appear clinically benign," European Journal of Surgical Oncology, 2015.

[44] J. J. Smith, O. S. Chow, M. J. Gollub et al., "Organ preservation in rectal adenocarcinoma: a phase II randomized controlled trial evaluating 3-year disease-free survival in patients with locally advanced rectal cancer treated with chemoradiation plus induction or consolidation chemotherapy, and total mesorectal excision or nonoperative management," BMC Cancer, vol. 15, article 767, 2015. 


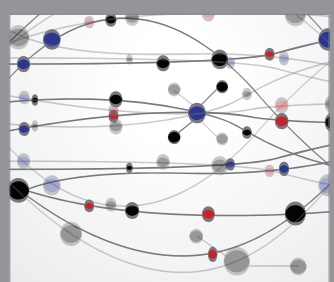

The Scientific World Journal
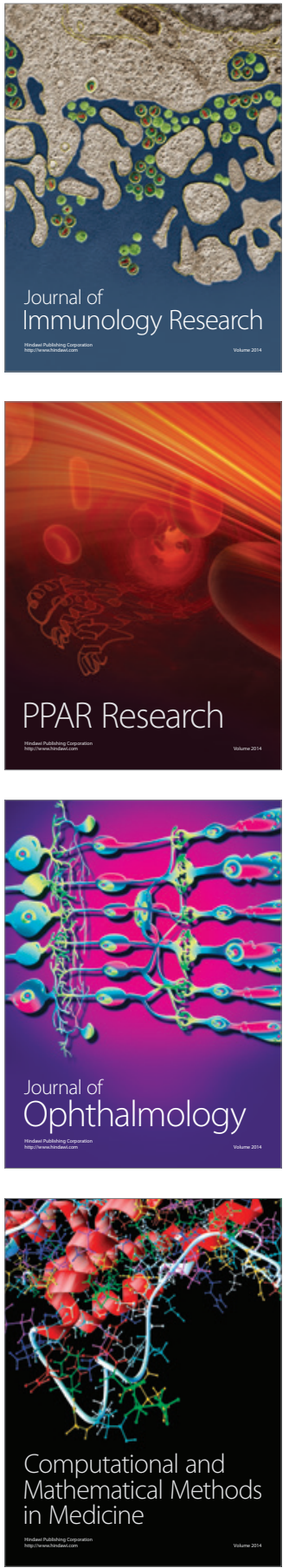

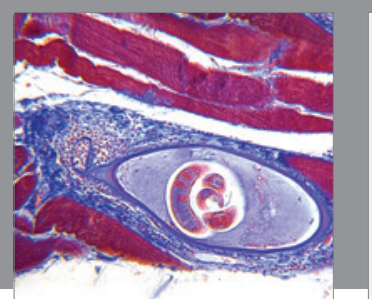

Gastroenterology Research and Practice

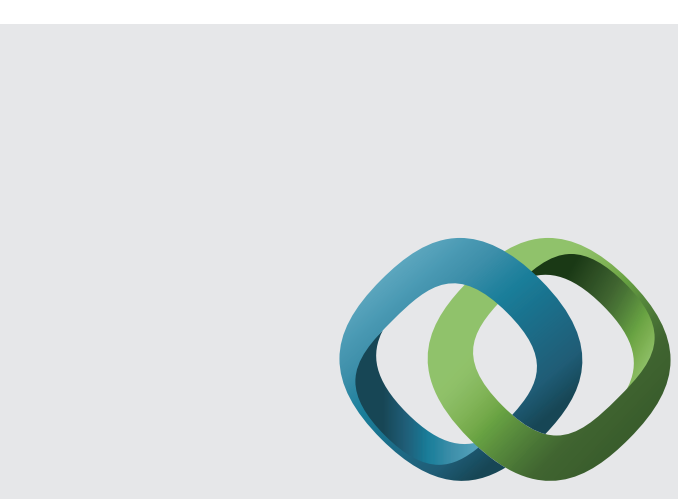

\section{Hindawi}

Submit your manuscripts at

http://www.hindawi.com
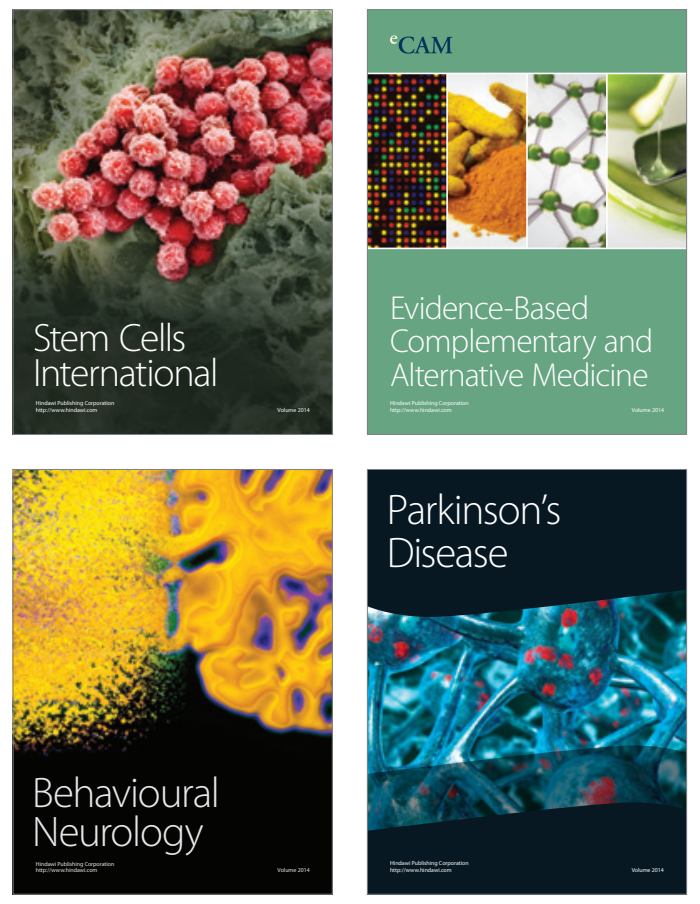
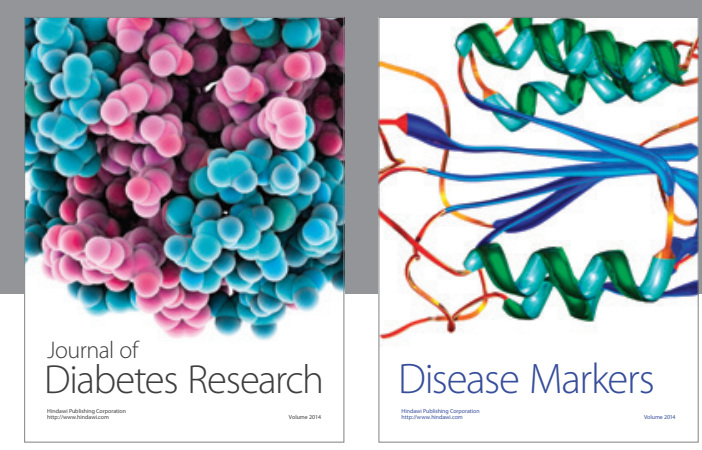

Disease Markers
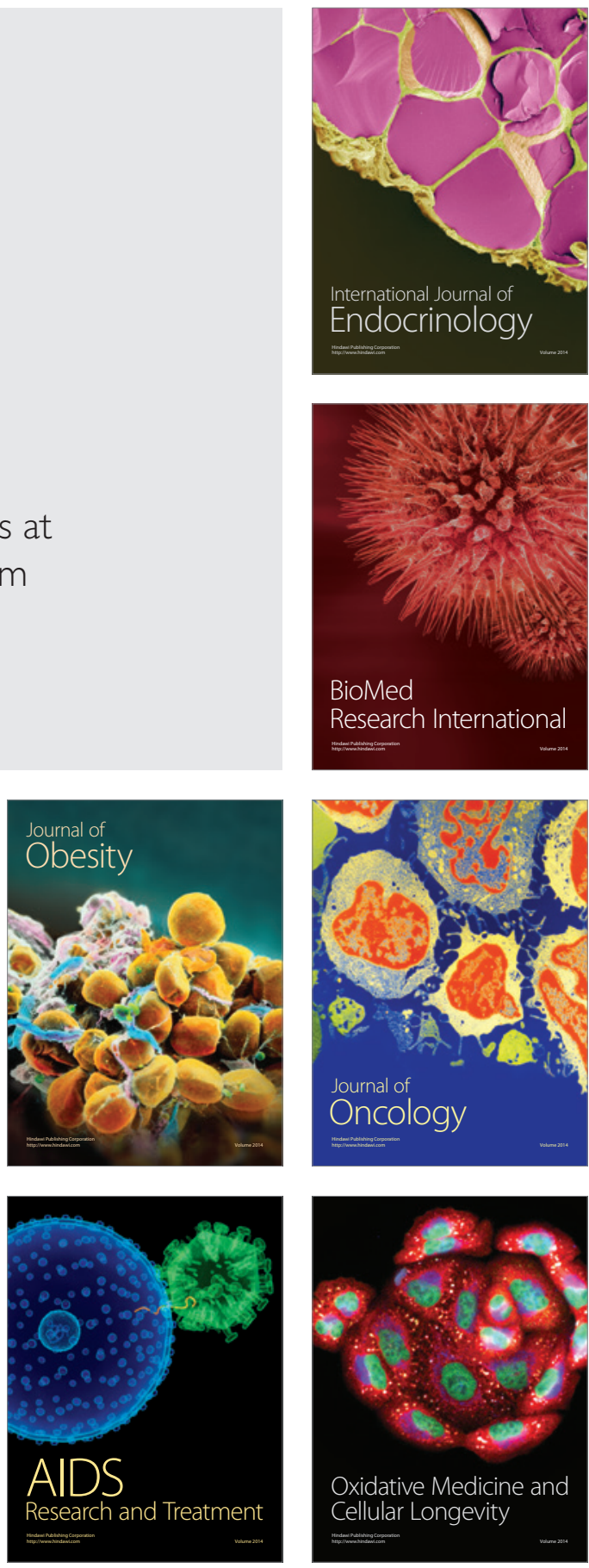